\title{
Phase transformation via a monoclinic phase in relaxor-based ferroelectric crystal $\left(\mathrm{PbMg}_{1 / 3} \mathrm{Nb}_{2 / 3} \mathrm{O}_{3}\right)_{1-x}\left(\mathrm{PbTiO}_{3}\right)_{x}$
}

\author{
Chi-Shun Tu, ${ }^{1, *}$ V. Hugo Schmidt, ${ }^{2}$ I.-C. Shih, ${ }^{1}$ and R. Chien ${ }^{2}$ \\ ${ }^{1}$ Department of Physics, Fu Jen University, Taipei, Taiwan 242, Republic of China \\ ${ }^{2}$ Department of Physics, Montana State University, Bozeman, Montana 59717
}

(Received 22 August 2002; published 24 January 2003)

\begin{abstract}
A monoclinic phase is evidenced between rhombohedral and cubic phases in a $\langle 111\rangle$-cut single crystal $\left(\mathrm{PbMg}_{1 / 3} \mathrm{Nb}_{2 / 3} \mathrm{O}_{3}\right)_{0.67}\left(\mathrm{PbTiO}_{3}\right)_{0.33}(\mathrm{PMN}-33 \% \mathrm{PT})$ from observation of domain structures. Near $360 \mathrm{~K}$ the structure begins to distort from the rhombohedral toward the tetragonal phase through monoclinic domains (probably $M_{A}$ type but perhaps $M_{B}$ type). However, the present $\langle 111\rangle$-cut crystal seems to disfavor the tetragonal phase and persists in the monoclinic phase up to $T \sim 420 \mathrm{~K}$, where the cubic phase begins to develop. Temperature-dependent orientations of optical indicatrices of domains indicate polarization rotations within the monoclinic planes. In addition, a previously electric-field-cooled process enhances a long-range transformation.
\end{abstract}

DOI: 10.1103/PhysRevB.67.020102

Relaxor-based ferroelectric (FE) crystals $\left(\mathrm{PbMg}_{1 / 3} \mathrm{Nb}_{2 / 3} \mathrm{O}_{3}\right)_{1-x}\left(\mathrm{PbTiO}_{3}\right)_{x} \quad(\mathrm{PMN}-x \mathrm{PT}) \quad$ and $\left(\mathrm{PbZn}_{1 / 3} \mathrm{Nb}_{2 / 3} \mathrm{O}_{3}\right)_{1-x}\left(\mathrm{PbTiO}_{3}\right)_{x}(\mathrm{PZN}-x \mathrm{PT})$ have a morphotropic phase boundary (MPB) between rhombohedral $(R)$ and tetragonal $(T)$ phases in the range $0.28 \leqslant x \leqslant 0.36$ and $x$ $<0.15$, respectively. ${ }^{1,2}$ However, these phase transformations that are unlike normal FE phase transitions exhibit apparent spatial inhomogeneity, and dependence on external $E$-field history and crystallographic orientation. ${ }^{3,4}$ Durbin et al. proposed, by in situ x-ray diffraction, that under $E$ field along $\langle 001\rangle$ PZN-8\%PT can no longer be perfectly rhombohedral, but that instead it is almost certainly monoclinic $(M){ }^{5}$ From the $E$-field-dependent polarization, a metastable orthorhombic $(O)$ FE phase (between $R$ and $T$ phases) was proposed in PZN-8\%PT crystals oriented along $\langle 001\rangle,\langle 110\rangle$, and $\langle 111\rangle^{6}$ The unit-cell size of this intermediate $O$ phase is double that of the simple unit cell that has $M$ symmetry. By synchrotron x-ray diffraction, an $O$ phase was observed in the PZN- $x$ PT system for $x=8 \%$ and $x=9 \%$ after a prior $E$-field application along $\langle 001\rangle .^{7} \mathrm{PZN}-9 \% \mathrm{PT}$ even shows the $O$ phase without a prior $E$-field poling. This $O$ phase was found in a narrow range $9 \% \leqslant x \leqslant 10 \% .^{7}$ On the other hand, a new lowersymmetry phase of monoclinic $M_{A}(Z=2$ formula units per unit cell) type (space group $\mathrm{Cm}$ ) was found in the $\mathrm{PbZr}_{1-x} \mathrm{Ti}_{x} \mathrm{O}_{3}$ (PZT) system for $0.52 \geqslant x \geqslant 0.46 .{ }^{8}$

By optical investigation, a rhombohedral/monoclinic $(R / M)$ phase coexistence was proposed at room temperature for a $\langle 001\rangle$-cut PMN-33\%PT crystal. $^{9}$ With a prior $E$-field poling along a $\langle 011\rangle$-cut PMN-33\%PT crystal, an extra $O$ phase between $R$ and $T$ phases was claimed from the dielectric result. ${ }^{10}$ Based on synchrotron x-ray diffraction, a $M_{A}$-type $M$ phase was observed at room temperature in a $\langle 001\rangle$-cut PMN-35\%PT single crystal previously poled under an $E$ field of $43 \mathrm{kV} / \mathrm{cm}^{11}$ However, unpoled and weakly poled PMN-35\%PT samples exhibit an average $R$ symmetry. ${ }^{11} \quad$ By neutron Rietveld analysis, a $M_{C}(Z=1)$-type (space group $\left.P m\right) M$ phase was evidenced in unpoled PMN-35\%PT powder samples at $80 \mathrm{~K} .{ }^{12}$ More recently, a $M_{C}$-type $M$ phase has been found in unpoled ceramics of PMN- $x$ PT for $37 \% \geqslant x \geqslant 31 \% .{ }^{13}$ Various phase co-
PACS number(s): 77.80.Dj, 77.80.Bh

existences of $R / M_{C}, T / M_{C}$, and $T / M_{C} / O$ at both 20 and $300 \mathrm{~K}$ were proposed for $31 \%, 33 \%$, and $35 \%$ PT compositions respectively. ${ }^{13}$ It is important to note that the effect of $M_{A}$-type polarization rotation (from $\langle 111\rangle$ to $\langle 001\rangle$ ) will be largely cancelled in randomly oriented ceramics. ${ }^{14}$

These phenomena open a different window in relaxorbased ferroelectrics. One may ask whether the intermediate phases $(M$ or $O)$ or various phase coexistences are intrinsic, or are merely due to spatial phase segregation, or fieldinduced strains of a given phase away from the ideal structure for that phase. In this report, polarizing microscopy was used to investigate temperature-dependent domain structures of a $\langle 111\rangle$-cut PMN-33\%PT single crystal.

The lead magnesium niobate-lead titanate crystal PMN$33 \% \mathrm{PT}$ was grown using a modified Bridgman method. ${ }^{15}$ The sample was cut perpendicular to the $\langle 111\rangle$ direction and the thickness is near $50 \mu \mathrm{m}$. A JEOL6100 electron microscope was used to determine concentrations of local $B$-site ions. The domain structures were observed by using a Nikon E600POL polarizing microscope with a crossed polarizeranalyzer pair. Transparent conductive films of ITO (indium tin oxide) were deposited on sample surfaces. The experimental configuration is illustrated in Fig. 1. For the zerofield-heated (ZFH) run, as shown in Fig. 2(a), domain structures were observed from $275 \mathrm{~K}$ up to the cubic phase without a dc bias field. In the field-cooled-zero-field-heated

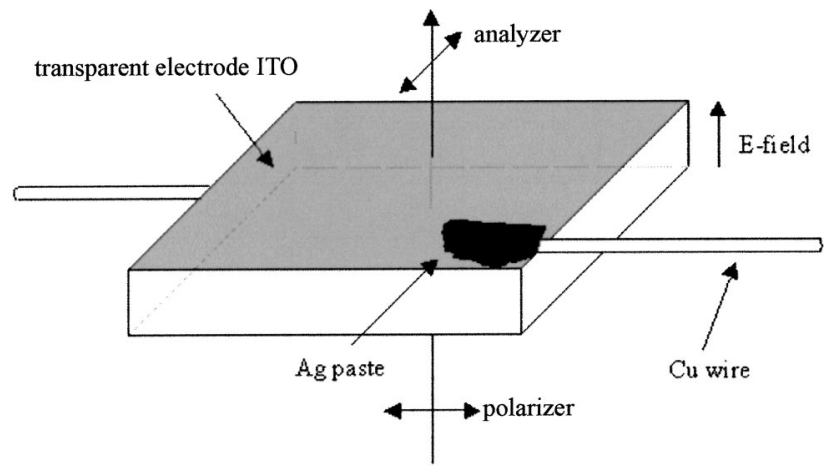

FIG. 1. The experimental configuration for observing domain structures under the polarizing microscope. A dc bias field was applied along $\langle 111\rangle$ during the field-cooled process. 


\section{(a) $\mathrm{ZFH}$}
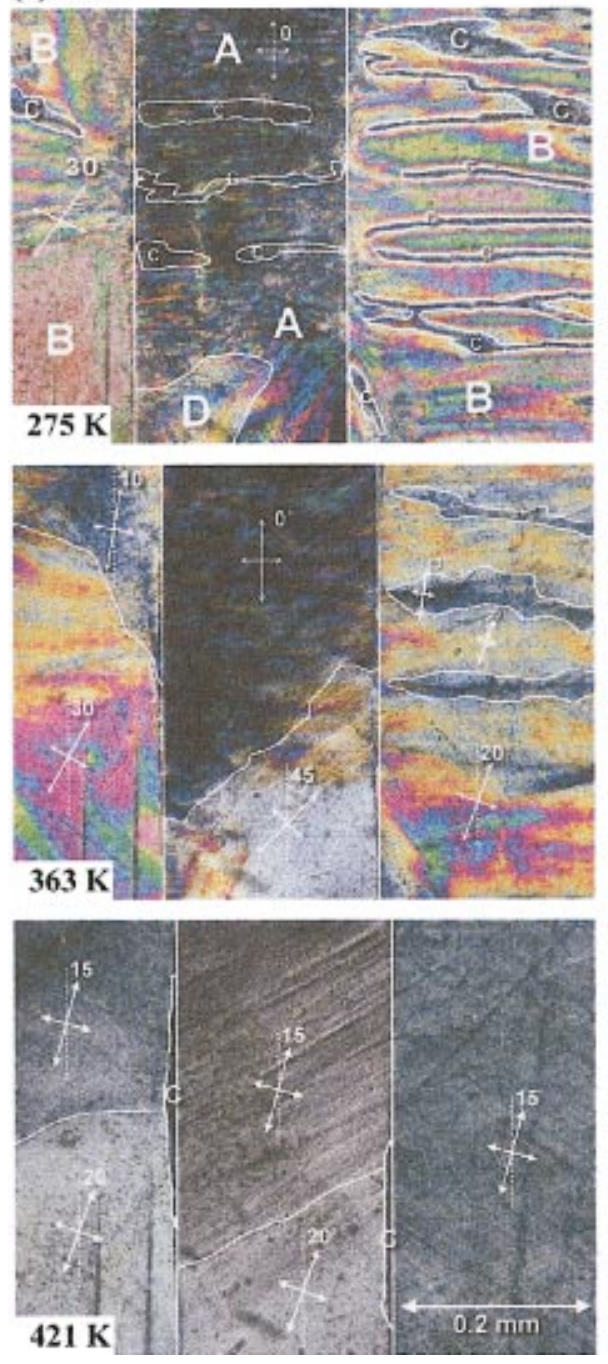

\section{(b) FC-ZFH}
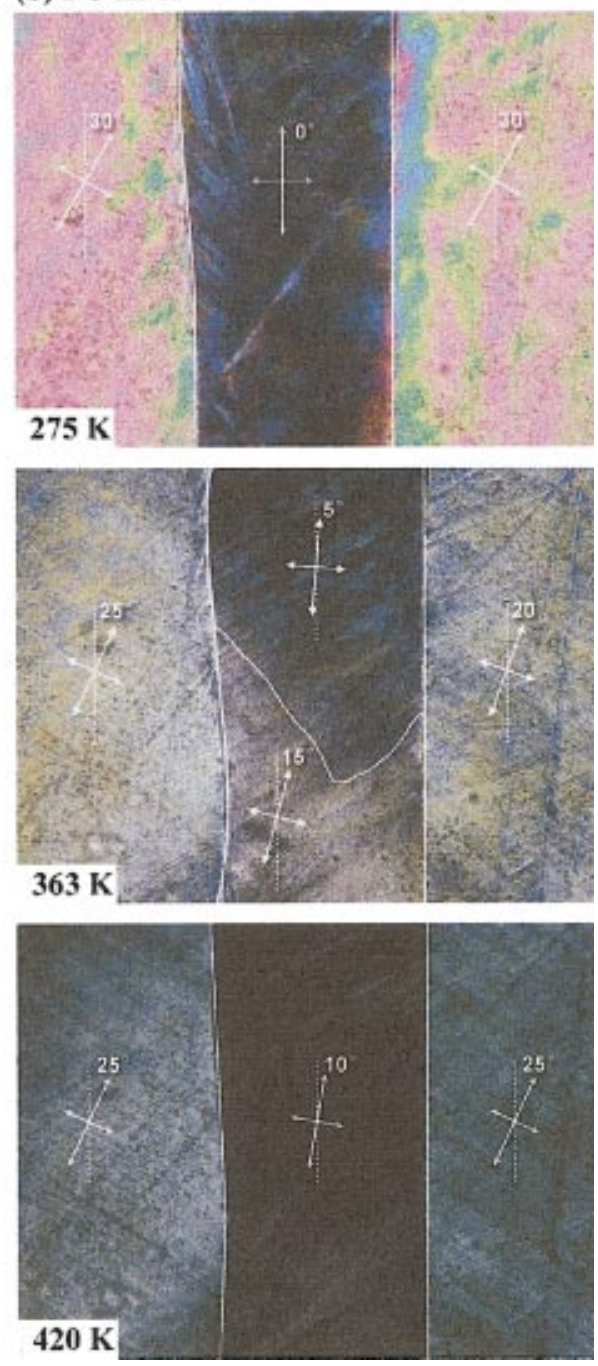

FIG. 2. (Color) Domain structures with various orientations of optical indicatrices observed from (a) ZFH and (b) FC-ZFH.
(FC-ZFH) run [Fig. 2(b)], the sample was first cooled from the cubic state down to $275 \mathrm{~K}$ with a dc bias field of $E$ $=8 \mathrm{kV} / \mathrm{cm}$ (which is greater than its coercive field $E_{c}$ $\sim 5 \mathrm{kV} / \mathrm{cm}$ at room temperature $)^{4}$ along $\langle 111\rangle$. Then, domain structures were observed upon heating without a bias field. In addition, as shown in Fig. 3, an observation under $E$ field of $12 \mathrm{kV} / \mathrm{cm}$ was carried out at room temperature. The

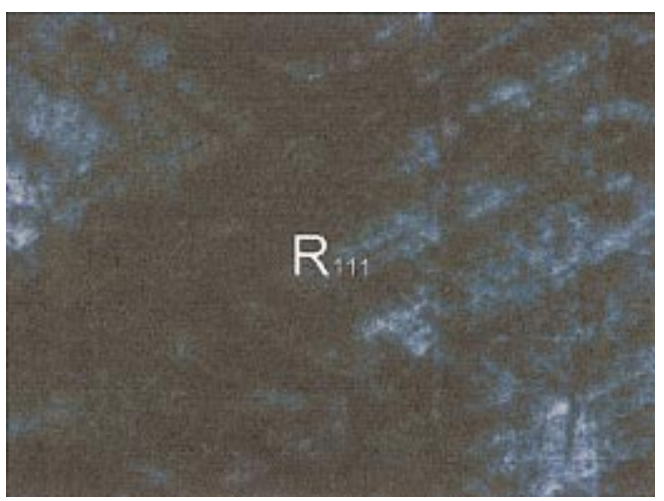

FIG. 3. (Color) Domain structures observed under $E$ $=12 \mathrm{kV} / \mathrm{cm}$ at room temperature. temperature-dependent dielectric permittivity is also given in Fig. 4.

A review of principles for interpreting polarizing microscope photographs may be helpful. The propagation direction $\vec{k}$ of the polychromatic "white" light is along $\langle 111\rangle$ for this work. The most information is obtained from observation of optical extinction, so we consider these conditions: (i) there must be no optical activity for the direction $\vec{k}$, (ii) if $\vec{k}$ is along an optical axis there is no transmission, (iii) if $\vec{k}$ is not along an optical axis, the incident $\bar{E}$ must lie along one of the two perpendicular axes (optical indicatrices) in the plane perpendicular to $\vec{k}$ for which the optical-frequency permittivity is maximum or minimum. For uniaxial crystals, the optical axis direction is known from crystal symmetry. For instance, in the $3 m$ rhombohedral cell, the optical axis parallels the threefold axis which is also the polar direction.

In considering whether experimental data evidence distinct phases, it is helpful to classify the strains for cells of various phases, relative to the parent cubic cell shape. The direction of FE polarization $\mathbf{P}$ is important because transitions between phase/domain combinations with similar $\mathbf{P}$ directions are more likely. Distortion to phases of lower symmetry (monoclinic or triclinic) allows $\mathbf{P}$ to vary continuously 


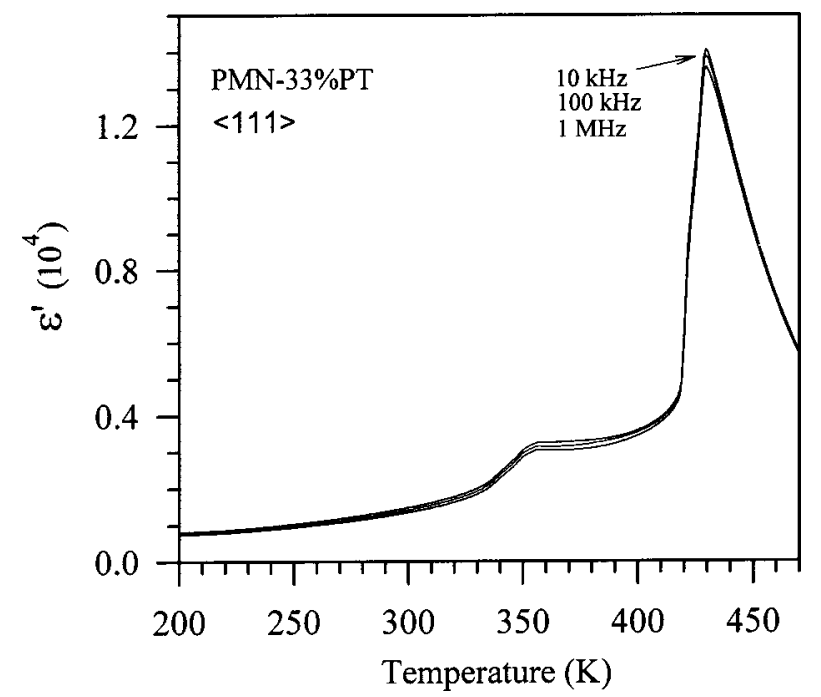

FIG. 4. Temperature- and frequency-dependent dielectric permittivity upon heating.

during the distortion. The $T$ and $R$ phases have uniaxial strain tensors with principal axes and polarization $\mathbf{P}$ along $\langle 001\rangle$ and $\langle 111\rangle$, respectively. For the $O$ phase observed in perovskites, the strain tensor is biaxial, with principal axes (for one example) along [101], [101] , and [010] and $\mathbf{P}$ along $\pm[101]$ or $\pm[10 \overline{1}]$. Monoclinic cell strain tensors are biaxial, with one principal axis along $\langle 001\rangle$ or $\langle 110\rangle$, and the other two axes and $\mathbf{P}$ in arbitrary directions perpendicular to that axis. The $\langle 001\rangle$ case corresponds to the cell based on the primitive unit cell $(Z=1)$ for the cubic phase, while the $\langle 110\rangle$ case corresponds to the cell based on the double-size orthorhombic cell $(Z=2)$.

Figure 5 shows the relations among the various phases and corresponding polarizations for primitive unit cell $(Z$ $=1$ ) and double-size orthorhombic cell $(Z=2)$. A rectangle indicates the directions of tetragonal polarization vectors $\mathbf{P}$. A triangle indicates directions for rhombohedral P's. A circle indicates directions for orthorhombic P's. Solid and dashed lines run along all edges and face diagonals, indicating directions that polarizations can take for monoclinic cells based on the double-size $(Z=2)$ orthorhombic cell. There are six planes corresponding to these lines. Dotted lines alternate between rectangles and circles, indicating directions that polarizations can take for monoclinic cells based on the simple $(Z=1)$ cubic, tetragonal, or rhombohedral cells. There are three planes corresponding to these lines. Any polarization whose direction does not correspond to one of the three symbol types or two types of lines results from a triclinic-shaped cell. Note that in most cases, neighboring symbols are joined directly by lines. This indicates that most higher-symmetry phases $(O, R$, or $T)$ with nearby polarization directions are related directly by monoclinic phases.

A series of $M$ cells can link two cells with nonarbitrary strain principal axes. For instance, cells with $\mathbf{P}$ along [001] and [101] are linked by $M_{C}$ cells with a principal axis $b_{m}$ along [010]. By extending this series of $M_{C}$ cells, one reaches a $T$ cell with $\mathbf{P}$ along [100]. Note that if one claims $T / O$ phase coexistence rather than just more or less distorted $T$ domains, one should see a marked preference for $\mathbf{P}$ along

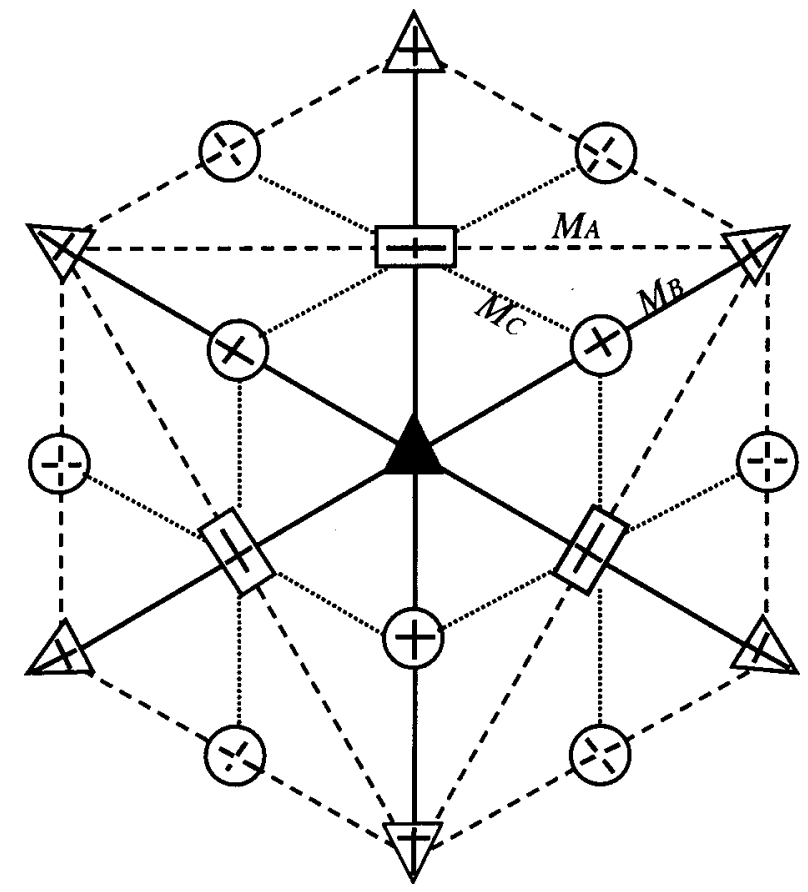

FIG. 5. The relations of various phases and corresponding polarizations. The meanings of various symbols and lines are described in the text.

[101] in the corresponding $O$ cell, rather than just a distribution of $M_{C}$ distortions away from $T$ domain cell shapes.

Similarly, $R$ cells with $\mathbf{P}$ along [111] and [1 111$]$ are linked by monoclinic $M_{B}$ cells with a principal axis $b_{m}$ along [101] , and midway along that linkage is the $O$ cell with $\mathbf{P}$ along [101]. Thus $R / O$ phase coexistence must be evidenced by marked preference for the cell with $\mathbf{P}$ along [101], and not just a distribution of $M$ distortions away from $R$ domain shapes. Note that the same monoclinic cell series, but now called $M_{A}$ cells, allows $\mathbf{P}$ rotation in the same plane, [111] through the $T$ cell with $\mathbf{P}$ along [010], ending at $\mathbf{P}$ along [111 1$]$ for another $R$ cell.

Figure 5 also presents a projection along the optical propagation direction [111] of domain polarization directions described in Fig. 2. Domains that are optically inactive will have extinction for optical electric field along the radial and circumferential axes indicated by solid crossed lines inside the symbols. Dashed crossed lines inside some circles indicate that extinction will not be quite complete because of optical activity for such $O$ domains. Solid lines between some symbols indicate no shift in extinction directions away from those in symbols connected by these lines, which represent some of the $Z=2 M$ cell polarization directions. Lines for the remaining $Z=2$ (dashed) and all $Z=1$ (dotted) $M$ polarization directions indicate a shift in extinction direction away from radial and circumferential, and incomplete extinction due to optical activity. The central triangle is black, to indicate extinction for any optical-field direction.

Temperature-dependent domain structures are shown in Figs. 2(a) and (b), respectively, for ZFH and FC-ZFH. At $T$ $=275 \mathrm{~K}$ [Fig. 2(a)], as marked by A, B, and C, the domain matrix exhibits three main orientations of optical indicatrices which can be determined by extinction positions. Hereafter, 
the orientation of the optical indicatrix of " $\mathrm{A}$ " regions at 275 $\mathrm{K}$ is chosen as zero degrees $\left(0^{\circ}\right)$. Note that all pictures in Fig. 2 are for polarizer/analyzer set at $0^{\circ} / 90^{\circ}$. "B" regions show extinction at the angle of $30^{\circ}$, hence are bright in Fig. 2. " $\mathrm{C}$ " regions (which present spindlelike morphology) exhibit extinction at all angles, indicating the rhombohedral phase with the polarization along [111]. However, the " $D$ " region does not show extinction at all, perhaps due to strong local strain distorting the index ellipsoid. When observing the [111]-cut sample along the [111] direction, between a crossed polarizer-analyzer pair, as shown in Fig. 5, the rotation angle of optical extinction between adjacent $R$ domains around

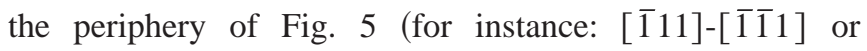
$[\overline{1} 11]-[1 \overline{1} 1])$ is $30^{\circ}$. To verify the phase in " $A$ " and " $B$ " regions, an observation under $E=12 \mathrm{kV} / \mathrm{cm}$ was carried out at room temperature (Fig. 3). The domain matrix exhibits mostly an angle-independent extinction, indicating a [111] rhombohedral phase as marked by $R_{111}$. The [111] rhombohedral domain should be induced easily by an external $E$ field on a [111]-cut crystal if the original phase is rhombohedral. Thus domains in " $A$ " and " $B$ " regions are certainly rhombohedral at $275 \mathrm{~K}$.

Orientations of optical indicatrices, as illustrated in Fig. 2(a), exhibit apparent changes near $360 \mathrm{~K}$ where the dielectric permittivity $\varepsilon^{\prime}$ (Fig. 4) shows a plunge accompanied by a slight frequency dispersion. As temperature increases, orientations of optical indicatrices exhibit gradual evolution up to angles of $15^{\circ}$ or $20^{\circ}$ near $420 \mathrm{~K}$. The cubic phase (as marked by " $\mathrm{C}$ ") begins to appear at $421 \mathrm{~K}$ as seen in Fig. 2(a). The crystal soon goes entirely into the isotropic cubic phase near $424 \mathrm{~K}$ which is consistent with $T_{m} \sim 425 \mathrm{~K}$ that corresponds to the maximum of $\varepsilon^{\prime}$.

What is this phase between 360 and $424 \mathrm{~K}$ ? We see no evidence for $O$ domains, because they would give the same $0^{\circ}$ and $30^{\circ}$ extinction angles as $R$ and $T$ domains, and we do not observe these extinction angles in this temperature range. This implies that the $O$ phase seen ${ }^{10}$ for prior $E$ field on a $\langle 011\rangle$-cut crystal of our PMN-33\% PT composition results only because that cut favors the $O$ phase when $E$ is applied. This lack of an $O$ phase in our crystal rules out the possibility that an $R$ domain transforms into an $M_{B}$ domain that brings it to an $O$ domain, after which a different $\left(M_{C}\right)$ monoclinic distortion brings it toward a $T$ domain. That is, the $M_{C}$ phase does not appear in our crystal. We note that the $M_{C}$ phase has so far been reported in PMN-PT only for powder ${ }^{12}$ and ceramic ${ }^{13}$ samples.

We cannot rule out the first part of the above process, that an $R$ domain transforms into an $M_{B}$ domain whose polarization rotates with increasing temperature toward that of a nearby hypothetical $O$ domain (see Fig. 5), but does not reach that direction before the crystal becomes cubic. However, Vanderbilt and Cohen ${ }^{16}$ found for PZT, by using Devonshire theory to eighth order of the order parameter, that $M_{B}$ regions are relatively small in order parameter space and may be harder to find in real systems than the $M_{A}$ phase.

The $M_{A}$ phase in our crystal would appear as a distortion of an $R$ domain toward a $T$ domain (see Fig. 5) as temperature increases above $360 \mathrm{~K}$, but the $T$ phase is not reached before the crystal becomes cubic at $424 \mathrm{~K}$. Additional support for our $M$ phase being $M_{A}$ instead of $M_{B}$ comes from first-principles calculations for $\mathrm{BaTiO}_{3}$ single crystal by $\mathrm{Fu}$ and Cohen, ${ }^{14}$ who found that the lowest free-energy path of polarization rotation is along the direct path $R-M_{A}-T$ instead of the path $R-M_{B^{-}} O-M_{C^{-}} T$ discussed above.

It appears from Fig. 5 and the temperature dependence of the domain patterns and extinction angles in Fig. 2 that for $\mathrm{ZFH}$ there is a rapid change above $360 \mathrm{~K}$ from an $R$ to an $M_{A}$ or possibly an $M_{B}$ phase. The polarization of that phase then changes further in direction in accord with the increase in extinction angle shift with temperature up to $424 \mathrm{~K}$.

In $\mathrm{FC}-\mathrm{ZFH}$, domain structures exhibit dramatic change in a narrow region of $\sim 360-365 \mathrm{~K}$, indicating a long-range phase transformation. Above $\sim 365 \mathrm{~K}$, the domain matrix exhibits a gradual evolution as temperature increases, and reaches a domain matrix near $420 \mathrm{~K}$ similar to that in the $\mathrm{ZFH}$ case. This reveals that the previous field-cooled process enhances a long-range transformation. However, the crystal still persists in an $M$ phase up to the cubic phase.

The authors would like to express sincere thanks to Dr. H. Luo for crystals. This work was supported by NSC Grant No. 91-2112-M-030-006 and DoD EPSCoR Grant Nos. N0001499-1-0523 and N00014-02-1-0657.
*Author to whom correspondence should be addressed. Electronic address: phys1008@mails.fju.edu.tw

${ }^{1}$ T. R. Shrout, Z. P. Chang, N. Kim, and S. Markgraf, Ferroelectr. Lett. Sect. 12, 63 (1990).

${ }^{2}$ J. Kuwata, K. Uchino, and S. Nomura, Ferroelectrics 37, 579 (1981)

${ }^{3}$ Z.-G. Ye and M. Dong, J. Appl. Phys. 87, 2312 (2000).

${ }^{4}$ C.-S. Tu, C.-L. Tsai, J.-S. Chen, and V. H. Schmidt, Phys. Rev. B 65, 104113 (2002).

${ }^{5}$ M. K. Durbin, E. W. Jacobs, J. C. Hicks, and S.-E. Park, Appl. Phys. Lett. 74, 2848 (1999).

${ }^{6}$ D. Viehland, J. Appl. Phys. 88, 4794 (2000).

${ }^{7}$ D. La-Orauttapong, B. Noheda, Z.-G. Ye, P. M. Gehring, J. Toulouse, D. E. Cox, and G. Shirane, Phys. Rev. B 65, 144101 (2002)
${ }^{8}$ B. Noheda, D. E. Cox, G. Shirane, R. Gao, B. Jones, and L. E. Cross, Phys. Rev. B 63, 014103 (2001).

${ }^{9}$ G. Xu, H. Luo, H. Xu, and Z. Yin, Phys. Rev. B 64, 020102(R) (2001).

${ }^{10}$ Y. Lu, D.-Y. Jeong, Z.-Y. Cheng, Q. M. Zhang, H. Luo, Z. Yin, and D. Viehland, Appl. Phys. Lett. 78, 3109 (2001).

${ }^{11}$ Z.-G. Ye, B. Noheda, M. Dong, D. Cox, and G. Shirane, Phys. Rev. B 64, 184114 (2001).

${ }^{12}$ J.-M. Kiat, Y. Uesu, B. Dkhil, M. Matsuda, C. Malibert, and G. Calvarin, Phys. Rev. B 65, 064106 (2002).

${ }^{13}$ B. Noheda, D. E. Cox, G. Shirane, J. Gao, and Z.-G. Ye, Phys. Rev. B 66, 054104 (2002).

${ }^{14} \mathrm{H}$. Fu and R. E. Cohen, Nature (London) 403, 281 (2000).

${ }^{15}$ H. Luo, G. Xu, P. Wang, and Z. Yin, Ferroelectrics 231, 97 (1999).

${ }^{16}$ D. Vanderbilt and M. H. Cohen, Phys. Rev. B 63, 094108 (2001). 